\title{
AN INVESTIGATION ON THE EFFECT OF CYCLIC DISPLACEMENT ON THE INTEGRAL BRIDGE ABUTMENT
}

\author{
Mojtaba MOVAHEDIFAR ${ }^{\mathrm{a}}$, Jafar BOLOURI-BAZAZ ${ }^{\mathrm{b}}$ \\ ${ }^{a}$ Department of Civil Engineering, Science and Research Branch, Islamic Azad University, Tehran, Iran \\ ${ }^{b}$ Department of Civil Engineering, Ferdowsi University of Mashhad, Mashhad, Iran
}

Received 04 Jun 2011; accepted 09 Aug 2012

\begin{abstract}
The integral bridge abutment, as a special type of retaining wall, is subject to cyclic displacement, which is due to the daily and seasonal temperature variations. The frame of this type of bridges is rigid and jointless. This requires that the slab of the bridge to be longitudinally continuous without expansion joints. This causes cyclic displacement to be imposed to the backfill material of integral bridge abutment. It should be pointed out that the omission of expansion joints helps to provide a fluent traffic and a reduction in maintenance and repair of the bridges. To investigate the impact of cyclic displacement on the loose backfill soil behaviour, an innovative laboratory retaining wall model has been designed and constructed to imitate the cyclic behaviour of backfill granular material. In addition, a numerical model, based on finite element method, has been developed to interpret the experimental results. This model was calibrated using the laboratory test data. The results indicate that the passive pressure, except for low amplitude displacement, escalates with progressive number of cycles and its distribution is not linear, which is due to the forming arch.
\end{abstract}

Keywords: integral bridge, retaining wall, cyclic, finite element, arching.

Reference to this paper should be made as follows: Movahedifar, M.; Bolouri-Bazaz, J. 2014. An investigation on the effect of cyclic displacement on the integral bridge abutment, Journal of Civil Engineering and Management 20(2): 256-269. http://dx.doi.org/10.3846/13923730.2013.802707

\section{Introduction}

Integral bridges abutment are jointless bridges where the superstructure is connected with the abutment. The rigid connection enables the abutment and superstructure to act as a single structural unit. This is due to the removal of expansion joints in the slab of integral bridges. This type of joints is widely used in the slab of traditional bridges (Khodair, Hassiotis 2005). The expansion joints in traditional bridges are the main reason for damages. Evaluation of cost maintenance, confirmed the joint removal in these bridges. (Marques Lima, de Brito 2009; Wallbank 1989; Ahn et al. 2011; Charuchaimontri et al. 2008). The consequence of the joint removal is that the bridge deck to be continuous. The daily and seasonal variations of temperature result in thermal expansion and contraction of deck (Arsoy 2000; Duncan, Arsoy 2003; Kim, Laman 2010a). This, in turn, results in imposition of cyclic horizontal displacements to the backfill soil of the abutments (Darley et al. 1998; Dicleli, Albhaisi 2004; Hoppe, Gomez 1996).

Figure 1 shows imposition of cyclic movement to the integral bridge deck to the wall abutment. If the ambient temperature and coefficient of thermal expansion of the bridge are $\delta_{T E B}$ and $\alpha$ respectively, the change in the length of the bridge, $d_{o}$, can be calculated as (Dicleli, Albhaisi 2004; Emerson 1977):
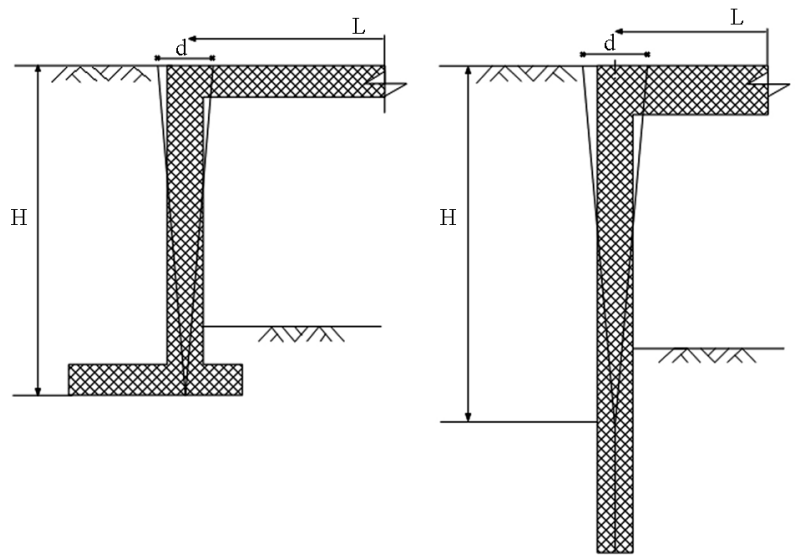

Fig. 1. Imposition of cyclic movement of integral bridge deck to the wall abutment

$$
d_{o}=\alpha \cdot \delta_{T E B} \cdot L,
$$

in which $L$ is the length of bridge deck (Fig. 1).

In practices, the soil at the back of the bridge abutment would resist against deck elongation. The actual bridge deck elongation is, therefore, less than $d_{o}$. Dicleli (2000) showed that the actual bridge deck elongation, $d$, could be calculated as (Fig. 1): 


$$
d=d_{o}-d_{c},
$$

in this relation $d_{c}$ is the amount of deck contraction due to the backfill material resistance (Dicleli 2000). Obviously, $d_{c}$ is depended to the bridge axial stiffness. However, the effect of backfill soil resistance on behaviour of the abutment wall movement is mostly neglected (Alizadeh et al. 2010).

The integral bridge abutments are generally analysed in plane strain condition. This assumption is reasonable since the abutment wing walls provide restraint of the soil transversely. If any out-of-plane deformation occurs, a plane strain analysis will overestimate the earth pressure (Phillip et al. 2010). Figure 2 shows the schematic view of an integral bridge abutment.

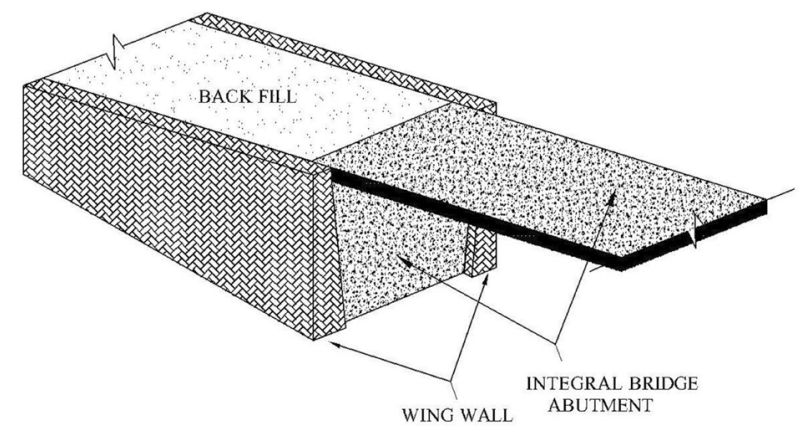

Fig. 2. A schematic view of integral bridges abutment

In spite of a lot of numerical and experimental works in passive pressure evaluation, the behaviour of this type of bridges has not already been well defined (Dicleli, Erhan 2010; Erhan, Dicleli 2009; Fang et al. 1994, 2002; Fang, Ishibashi 1986; James, Bransby 1970; Kim, Laman 2010b; Noorzaei et al. 2010).

A wide range of experimental research deals with investigation on the induced lateral earth pressure due to the cyclic movement of bridge deck (Barker, Carder 2001, 2000; Broms, Ingleson 1971, 1972; Card, Carder 1993; Horvath 2000; Sanford, Elgaaly 1993; Springman et al. 1996; Thomson 1999; Tsang et al. 2002).

Springman et al. (1996), for example, performed a series of tests on a smooth and rigid wall. The wall of their laboratory model was able to rotate about a hinge located at its bottom. The induced stress on the face of the wall was measured using the miniature pressure transducer. Their results indicate an increase in lateral earth pressure due to the cyclic movement. They also performed more tests to investigate the effect of wall rigidity on the pressure escalation. They recommended different magnitudes of the lateral earth pressure coefficients for rigid and flexible walls. The results indicate this coefficient for rigid wall is greater than that for flexible wall.

Barker and Carder (2001) performed in-situ tests in which pressure escalation behind a bridge abutment was measured. The length of the bridge was $40 \mathrm{~m}$ and lateral movement and stress behind the bridge abutment were evaluated. They observed a trend of the pressure increase during daily and seasonal temperature variations. Again, they evaluated stress variations of two bridges abutment.
The pressure and movement of the abutments were recorded during construction and the first year of operation. The results indicate that lateral earth pressure during construction, which was around at rest pressure, increased gradually afterwards. More investigations indicate that the lateral earth pressure distribution is not linear and reaches its maximum value near to the middle of abutment (Barker, Carder 2000).

England and Bolouri-Bazaz (1995) indicate that stress increases with depth initially and decreases gradually in the region adjacent to the wall bottom. This phenomenon can be well interpreted by forming arch in this region. The stress reduction due to the arching effect has been reported by many other researchers (Chen et al. 2011; Paik, Salgado 2003; Roberts 2010).

\section{Research significance}

The main objective of this research is to investigate the influences of cyclic displacement on the behaviour of sandy soil behind the abutment bridge. The present research has been mainly divided into two parts:

1) An adoption a numerical model to predict the pressure distribution on the face of a retaining wall, subject to the cyclic displacement. The finite element approach has been adopted to predict the performance of the backfill material and the lateral pressure escalation on the wall face;

2) Development a laboratory retaining wall model in order to validate the numerical model results. In addition, the strategies adopted by the Authors to carry out and interpret laboratory test results are included.

To achieve the above objectives, the Authors designed a laboratory wall model. Since the integral bridge abutments are generally analysed in plane strain condition and in practice, the abutment is mostly built in reinforced concrete, it is relatively rigid (Alizadeh et al. 2010). This is the reason that the retaining wall model was designed in such a way to maintain the plane strain condition. In addition, the wall itself was made from hard plastic and stiffened by backing alloys, to increase its rigidity. This facilitated to apply cyclic movement to the backfill material. In addition, the previous research clearly indicates that the effect of seasonal temperature variations and combination of daily-seasonal temperature variations are approximately identical (England et al. 2000). This is the reason that the temperature combination has not been considered in the present study.

The specifications of the laboratory model are presented in the following sections.

\section{Backfill material properties}

Firooz-Kouh sand with a similar gradient, texture and colour to Leighton Buzzard sand, was used in the current research. This sand was poured into the experimental device from a constant drop height of $100 \mathrm{~mm}$. Figure 3 shows the Firooz-Kouh gradient. 


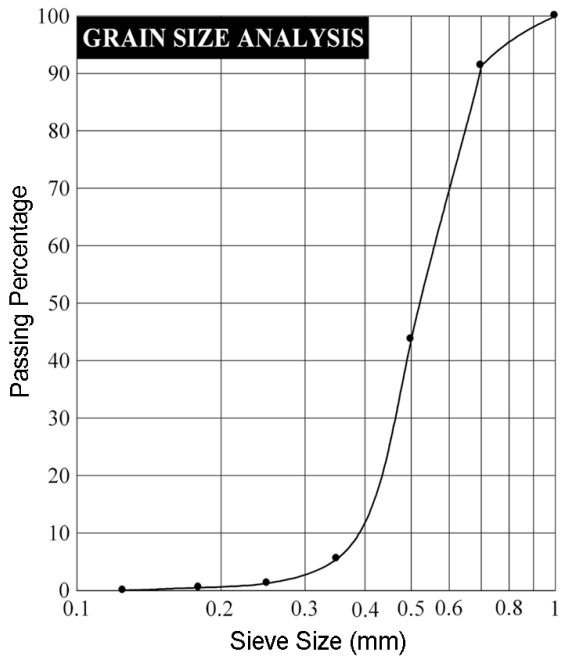

Fig. 3. Particle size distribution of Firooz-Kouh sand

The friction angle $(\phi)$ of this sand and friction angle between sand and wall using Triaxial and Direct Shear tests respectively (ASTM D4767, D3080). Due to the smoothed face of the wall, the friction angle between the walls and sand, which was measured by Direct Shear device, was very low and nearly to zero. The specifications of backfill material are gathered in Table 1.

Table 1. Specifications of Firooz-Kouh sand

\begin{tabular}{ccc}
\hline Unit weight & $\gamma$ & $13.92\left(\mathrm{kN} / \mathrm{m}^{3}\right)$ \\
Friction angle & $\phi$ & $32.5^{\circ}$ \\
Cohesion & $C$ & $0\left(\mathrm{kN} / \mathrm{m}^{2}\right)$ \\
Elasticity modulus & $E$ & $1.64 \times 10^{4}\left(\mathrm{kN} / \mathrm{m}^{2}\right)$ \\
Specific gravity & $G_{s}$ & 2.71 \\
Relative density & $D_{r}$ & $27 \pm 2 \%$ \\
Poisson's ratio & $v$ & 0.3 \\
\hline
\end{tabular}

\section{Numerical model}

To investigate the behaviour of sandy soil behind the bridge abutment, the Authors have developed a numerical model. The following section is a brief description of the numerical model.

\subsection{Model specifications}

The length and height of the developed numerical model were considered $1900 \mathrm{~mm}$ and $800 \mathrm{~mm}$ respectively. The numerical model dimensions were the same as in the experimental device, which has been described in the next section. Figure 4 illustrates the developed finite element numerical model in the present research.

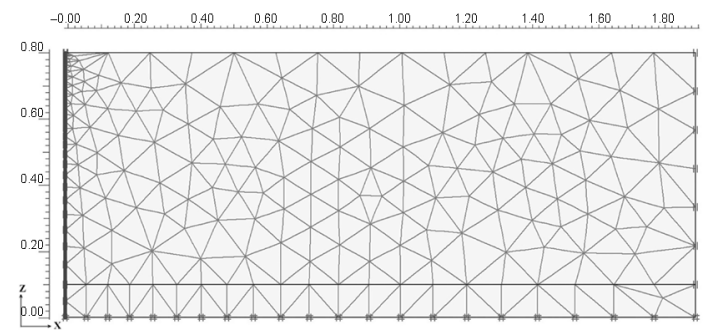

Fig. 4. Finite element numerical model
The hinge, around which the wall rotates, is located $100 \mathrm{~mm}$ above the bottom of the wall. The finite element, as a numerical method, was used to investigate the effects of cyclic displacement on the backfill material of the retaining walls.

\subsection{Soil modelling}

Soil is a complicated material, which behaves nonlinearly and often shows anisotropic and time dependent behaviour when subjected to stress. Various constitutive models have been proposed by several researchers to describe this nonlinearity behaviour. Several researchers discuss different models such as Mohr-Coulumb, Drucker-Prager, Duncan-Chang, Cam Clay, Soft Soil and Hardening Soil.

Olmo Segovia (2006) studied the behaviour of backfill material behind a steel box culvert. He indicated that in integral bridge abutments, the Mohr-Coulumb model in comparison with hardening model provides better correspondence to the field measurements. In addition, many researchers have used Mohr-Coulumb model for analysis of integral bridge abutments (Banks et al. 2008; Carlstedt 2008). It is evident that the hardening soil model may lead to more accurately results, but more parameters are required and makes it complicated.

The Mohr-Coulumb is an implicit model with five parameters, which can be easily and accurately determined in soil lab. In general, stress state, the model's stress-strain behaves linearly in the elastic range, with two defining parameters from Hooke's law (E and $v$ ). In addition, there are two parameters, which define the failure criteria (the friction angle, $\phi$. and cohesion, c) and a dilatancy angle, $\psi$. which to describe the flow rule. The dilatancy angle $(\psi)$ is normally used in cohesion-less materials and is dependent on the relative density and friction angle of the soil. For a soil material with friction angle greater than $30^{\circ}$, the soil tends to dilate at small strain conditions. The dilatancy used in the numerical model is therefore considered approximately equal to $\phi$ $30^{\circ}$ (Bolton 1986).

\subsection{Model formulation}

In plane strain, one deals with a situation in which the deformation of the structure in two directions, say the $x$ and $\mathrm{z}$ coordinate directions, is large in comparison with the third directions (y-coordinate). In other words the geometry of the structure is in such a way that the deformation in one direction is essentially zero or very small and negligible. One of the important practical applications of this situation occurs in the analysis of integral bridge abutment (Phillip et al. 2010).

In the formulation of material model in plane strain condition, the stress and strain tensors must be modified. The stress tensor can be represented by a matrix with nine components. Since the stress tensor is symmetric, i.e. $\sigma_{x y}=\sigma_{y x}, \sigma_{y z}=\sigma_{z y}$ and $\sigma_{z x}=\sigma_{x z}$, it involves only six components: 


$$
\bar{\sigma}=\left(\begin{array}{llllll}
\sigma_{x x} & \sigma_{y y} & \sigma_{z z} & \sigma_{x y} & \sigma_{y z} & \sigma_{z x}
\end{array}\right)^{T}
$$

in plane strain condition, however:

$$
\tau_{y x}=\tau_{y z}=0 .
$$

Similarly, the strain tensor is symmetric, it involves only six components, i.e.:

$$
\bar{\varepsilon}=\left(\begin{array}{llllll}
\varepsilon_{x x} & \varepsilon_{y y} & \varepsilon_{z z} & \gamma_{x y} & \gamma_{y z} & \gamma_{z x}
\end{array}\right)^{T}
$$

Again in plane strain condition:

$$
\varepsilon_{y y}=\gamma_{y x}=\gamma_{y z}=0 .
$$

Based on the above formulation the proposed model has been modified for plane strain condition.

In this model, according to the classical theory of plasticity, the total strain can be decomposed into elastic and plastic parts when the stress state reaches the yield surface, i.e.:

$$
\{d \varepsilon\}=\left\{d \varepsilon^{e}\right\}+\left\{d \varepsilon^{p}\right\} .
$$

The Hooke's law relates the stress and elastic strain increments as follows:

$$
\{d \sigma\}=\left[E^{e}\right]\left\{d \varepsilon^{e}\right\},
$$

by using of Eqn (7) and Eqn (8) we have:

$$
\{d \sigma\}=\left[E^{e}\right]\left(\{d \varepsilon\}-\left\{d \varepsilon^{p}\right\}\right) .
$$

In general, the plastic strain increment is written as following the normality rule:

$$
\left\{d \varepsilon^{p}\right\}=\lambda\{\partial g / \partial \sigma\} .
$$

where: $\lambda$ is a scalar plastic multiplier that can be calculated by Forward Euler's method (Smith, Griffiths 1998); and $g$ is the plastic potential function. According to Forward Euler's method and Mohr-Coulumb model:

$$
\lambda=\frac{\{\partial f / \partial \sigma\}\left[E^{e}\right]}{\{\partial f / \partial \sigma\}\left[E^{e}\right]\{\partial g / \partial \sigma\}}\{d \varepsilon\} .
$$

Substitute Eqn (11) and Eqn (10) into Eqn (9):

$$
\{d \sigma\}=\left(\left[E^{e}\right]-\frac{\left[E^{e}\right]\{\partial g / \partial \sigma\}\{\partial f / \partial \sigma\}\left[E^{e}\right]}{\{\partial f / \partial \sigma\}\left[E^{e}\right]\{\partial g / \partial \sigma\}}\right)\{d \varepsilon\},
$$

where $f$ is yield function and $g$ is plastic potential function.

It is appreciated that the Mohr-Coulumb is elastic perfectly plastic. This model is applicable for the first loading. For the second and subsequent cycles, the mesh is updated and the stiffness matrix is progressively modified. This model, in turn, is therefore updated which means that the model may be considered as a non-linear one.

It is well known that the stiffness of real soils depends significantly on the stress level. This means that the stiffness, which is generally referred as deformation modulus, can increases with depth. In the developed nu- merical model, it is possible to specify a stiffness that varies with depth. Since the height of the laboratory model is short, the magnitude of deformation modulus has been considered constant.

\subsection{Computational algorithm}

This numerical model is a 2-D finite element program designed to solve plane strain problems. The Figure 5 shows schematically the flow chart of the FE developed code.

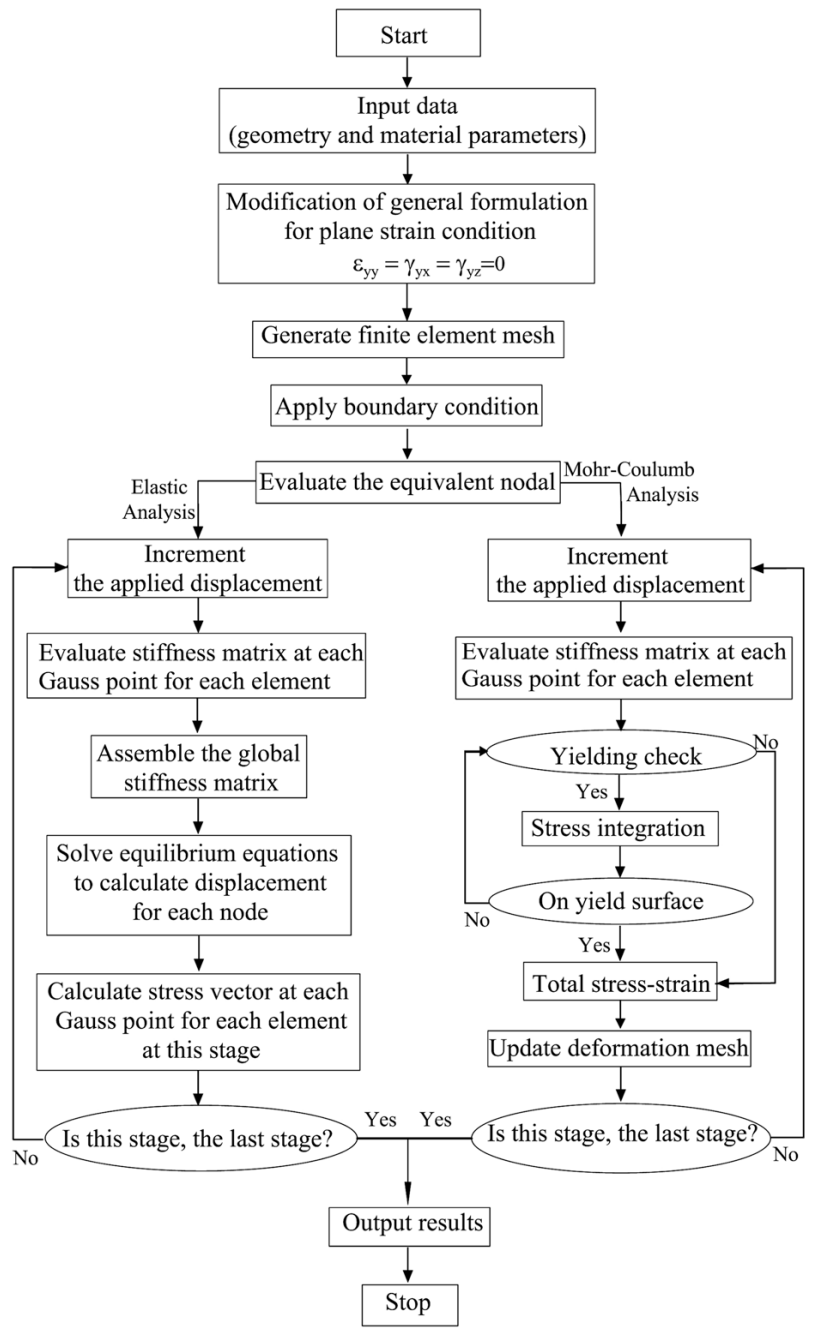

Fig. 5. A schematic flow chart for numerical model

This program discreteness the soil mass and employs triangular element with 6 and 15 -nodes. This code has a simple mesh generator to define the distribution of elements for each problem. The program provides soil, wall and interface elements.

Since in the laboratory model, the wall is completely rigid, it must be considered in the numerical model as well. To maintain wall rigidity, a linear displacement was applied to the wall in the numerical model. The applied displacement (which is equal to $d / 2$ ), varies linearly from the top to the bottom of the wall. This means the applied displacement reaches to zero at the hinge (Fig. 1). This ensures that no nonlinear deformation occurs in the wall element in the model, i.e. the wall remains totally rigid. 
For soil-structural interaction situation, such as retaining wall, relative deformation of wall with respect to the soil must be properly modelled. A continuum element which satisfies deformation compatibility between soil and structure should be considered in the numerical model. An interface element is used to model this situation. Many methods including use of thin continuum element, linkage element, Hybrid method and zero or finite thickness element have been proposed to model discontinuous behaviour at the soil-structure interface (Potts, Zdravkovic' 2001). However, the zero or finite thickness method has been employed in the current research. These elements allow defining reduced/increased strength parameters between the two different types of elements. Thin rectangular interface element with six nodes was used between soils and structural elements. The interface element had a zero thickness in the finite element formulation. However, a small virtual thickness was assigned to the interface element, which was used to define the material properties of the interface. The material properties of the interface element were the same as those of surrounding soil elements, except that a strength reduction factor $\left(R_{\text {inter }}\right)$, defined as the ratio of the interface strength to the shear strength of surrounding soils, was used for the interface element.

The properties of interface element are calculated from the soil properties, i.e.:

$$
\begin{gathered}
c_{i}=R_{\text {int er }} c_{\text {soil }} ; \\
\tan \phi_{i}=R_{\text {int er }} \tan \phi_{\text {soil }} \leq \tan \phi_{\text {soil }} .
\end{gathered}
$$

In general, for real soil-structure interaction the interface is weaker and more flexible than associated soil layer, which means that value of $R_{\text {inter }}$ should be less than 1 (Das 2002). However, since the interface is considered smooth, this parameter has been set to be as low as possible, 0.1 .

\section{Specifications of the experimental device}

The dimensions of the experimental device are $400 \mathrm{~mm}$ wide and $850 \mathrm{~mm}$ high, allowing soil depth up to $800 \mathrm{~mm}$ to be filled. The length of the apparatus is long enough to allow the rupture surface to be formed wholly. Based on the maximum friction angle of the backfill sand which measured as $\phi=40^{\circ}$ and assuming plane rupture surface with angle of $45+\phi / 2$ with respect to vertical and $H=700 \mathrm{~mm}$, the length of the apparatus, $L$, has been calculated as:

$$
L=H \cdot \tan (45+\phi / 2) \approx 1500 \mathrm{~mm} .
$$

Since the rupture surface is not completely plane and tends to be curvature, the length of the laboratory model is increased to $1900 \mathrm{~mm}$. The frame of the model wall was made from steel. In addition, the rigid retaining wall itself was made from hard plastic material and stiffened by backing alloys, to increase its rigidity. This prevents deformity when applying load. The wall could rotate about its toe by a hinge, which was installed in $100 \mathrm{~mm}$, measured from the bottom of the apparatus res- ervoir bed. This resulted in up to $700 \mathrm{~mm}$ soil depth to be contributed in test. The top wall displacement is achieved by the aid of mechanical motor. The driving shaft of the motor provides inward and outward displacement to simulate cyclic displacement.

Soil stress measurements were made on the face of the wall using the pressure transducer with flat diaphragm, which was very sensitive to the lateral soil pressure. Six pressure transducers, PT1 to PT6, are installed at the height of $H=20,30,40,50,60$ and $70 \mathrm{~cm}$ measured from the bed of the sand reservoir (Fig. 6). In addition, in order to observe the soil deformation during the test, both longitudinal sides of the apparatus were made from the thick $(10 \mathrm{~mm})$ glass panel. In addition, the horizontal movements of the wall were captured; using two LVDTs installed at the top and the middle of the wall. This facilitated to measure the horizontal movement of the wall and to control its rigidity during the test. The reading of these LVDTs in all tests showed no wall bending occurred. Figure 6 illustrates a schematic presentation of the apparatus.

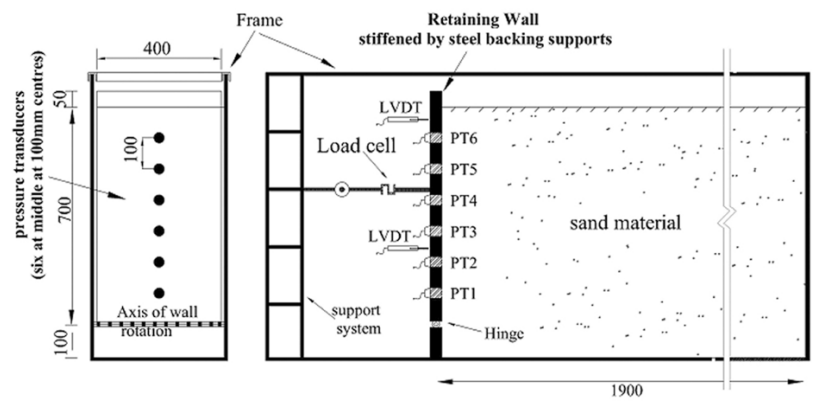

Fig. 6. A schematic view of the retaining wall model apparatus

Furthermore, shown in Figure 7 is a general view of the laboratory retaining wall model. As stated earlier, in practice, the behaviour of the integral bridge abutment is in plane strain condition. To maintain this condition in the laboratory model, the glassy sidewalls (wing walls in practice) were fixed inside a frame. This prevented the lateral movement of sidewalls. It, in turns, results in plane strain conditions to be maintained.

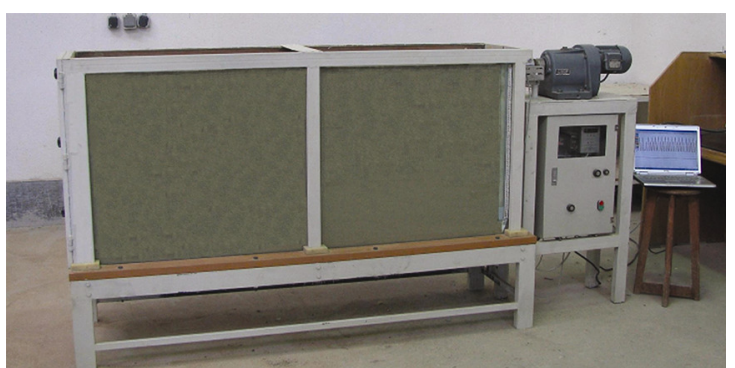

Fig. 7. A general view of the laboratory model apparatus

\section{Test program}

In the present research, a wide range of experimental tests has been carried out. The main purpose of these tests was to validate the numerical model results. Table 2 summarizes the details of all tests. 
Table 2. The test program

\begin{tabular}{|c|c|c|c|c|c|}
\hline $\begin{array}{l}\text { No. of } \\
\text { test } \\
\text { series }\end{array}$ & $\begin{array}{c}d / 2 \\
(\mathrm{~mm})\end{array}$ & $\begin{array}{c}d /(2 \mathrm{H}) \\
(\%)\end{array}$ & $\begin{array}{c}\text { Velocity } \\
(\mathrm{mm} / \mathrm{s})\end{array}$ & $\begin{array}{c}\text { Period } \\
\text { (s) }\end{array}$ & $\begin{array}{c}\text { Maximum } \\
\text { number of } \\
\text { Cycles }\end{array}$ \\
\hline 1 & 0.20 & 0.029 & $\begin{array}{c}0.015 \\
0.075 \\
0.15 \\
\end{array}$ & $\begin{array}{c}53.3 \\
10.6 \\
5.3 \\
\end{array}$ & 120 \\
\hline 2 & 0.50 & 0.071 & $\begin{array}{c}0.015 \\
0.075 \\
0.15 \\
\end{array}$ & $\begin{array}{c}133.3 \\
26.6 \\
13.3 \\
\end{array}$ & 80 \\
\hline 3 & 1.00 & 0.029 & $\begin{array}{c}0.015 \\
0.075 \\
0.15 \\
\end{array}$ & $\begin{array}{c}266.6 \\
53.3 \\
26.6 \\
\end{array}$ & 80 \\
\hline 4 & 1.75 & 0.029 & $\begin{array}{c}0.015 \\
0.075 \\
0.15 \\
\end{array}$ & $\begin{array}{c}466.6 \\
93.3 \\
46.6 \\
\end{array}$ & 80 \\
\hline 5 & 2.45 & 0.029 & $\begin{array}{c}0.015 \\
0.075 \\
0.15\end{array}$ & $\begin{array}{c}653.3 \\
130.6 \\
65.3 \\
\end{array}$ & 50 \\
\hline 6 & 6.50 & 0.029 & $\begin{array}{c}0.015 \\
0.075 \\
0.15 \\
\end{array}$ & $\begin{array}{c}1733.3 \\
346.6 \\
173.3 \\
\end{array}$ & 50 \\
\hline
\end{tabular}

In this table, the magnitudes of displacement, rotation, velocity of top wall movement, period and maximum number of cycles are gathered.

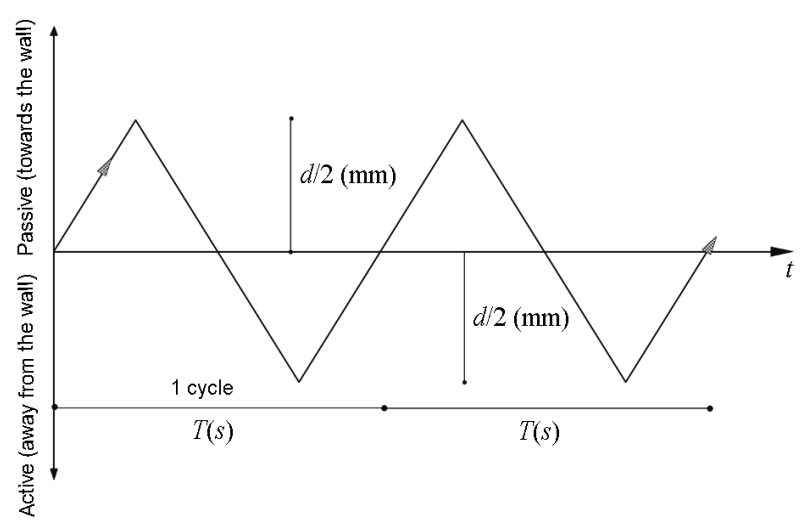

Fig. 8. The time-history of the applied displacement

In addition, the time-history of the applied displacement is illustrated in Figure 8.

The following sections describe the effects of the different velocity of wall movement, the magnitude of wall rotation and the number of cycles on the lateral passive pressure.

\section{Test results}

Based on the test program (Table 2) the experimental test results together with the numerical analysis are presented in the following sections.

\subsection{Effect of velocity magnitude}

In this Section, the effect of different velocity of the top of the wall movement on the lateral passive pressure in the numerical model is investigated. The rate of movement are including $v=0.015,0.075$ and $0.15 \mathrm{~mm} / \mathrm{s}$. To evaluate the numerical model results, cyclic tests were also conducted using the laboratory wall model, with various wall rotation including $(d / 2)_{\max }=0.2,0.5,1,1.75$, 2.45 and $6.5 \mathrm{~mm}(d / 2$ is top of the wall movement in passive condition; see Fig. 1). The six-pressure transducer installed on the wall face measured the stress escalation. Figures 9 and 10 show comparative the passive pressure escalation just for the first cycle for the numerical and laboratory models respectively. As shown, the rate of wall movement has no significant effect on the passive pressure magnitude. This may be due to the very low amplitude of velocity. In practice, of course, as we know, the rate of retaining wall abutment of the integral bridges is much less than these magnitudes (Darley et al. 1998). It should be pointed, carrying the tests in the laboratory with real velocity as in practice, takes a long time. Again, it emphasizes that the wall movement velocity has no significant effect on the magnitudes of passive earth pressure. It is worth mentioning that in a specific wall rotation, [e.g. $(d / 2)_{\max }=2.45 \mathrm{~mm}$ ], and in identical cycle, say cycle 50 , no significant difference between velocity of $v=0.015,0.075$ and $0.15 \mathrm{~mm} / \mathrm{s}$ was observed. This is the reason that data are shown just for the first inward movement of the wall.

Furthermore, illustrated in Figure 11 is the comparison among the computed passive earth pressures from the numerical model and the captured data from the laboratory model just for the wall movement velocity of $v=$ $0.075 \mathrm{~mm} / \mathrm{s}$ (the first cycle). Satisfactory agreement between the predicted and measured wall response was obtained.

As shown, the rate of wall movement has no significant effect on the passive pressure magnitude. This is the reason that the results reported here are just for the wall movement velocity of $v=0.075 \mathrm{~mm} / \mathrm{s}$.

\subsection{Effect of wall rotation}

The focus in the current section is on the magnitude of wall rotation on the lateral passive pressure. For the purpose, the top of the wall was horizontally pushed inward (passive condition) and pulled backward to the initial vertical condition and further to active condition and repeatedly. A wide range of the wall rotation, starting from vertical position, was considered to cover most practical situations, including $(d / 2)_{\max }=0.2,0.5,1,1.75$, 2.45 and $6.5 \mathrm{~mm}$, equivalent to the wall rotation $(\mathrm{d} / 2 \mathrm{H})$ of $0.029,0.071,0.143,0.25,0.35$ and $0.929 \%$ respectively. Theses value are selected in such a way to match with practical values and other researchers tests (Lock 2002).

Figure 11 depicts the induced lateral passive earth pressure on the face of the wall for the numerical and laboratory test models. The results are shown only for the first cycle. Comparison of the numerical analysis and experimental data shows about 10 percent deviation, which seems to be acceptable. The difference between the numerical and experimental results may be due to the assumptions considered in the numerical model. In the present research, for example, the soil behavioural which has been modelled with well known Mohr-Coulumb criterion, is considered to be as elastic perfectly plastic. The soil behaviour, however, does not exactly coincide with 
the model assumptions, which are essentially simplified. To explain the results more precisely, the numerical analysis results together with the pressure at rest $\left(k_{\mathrm{o}}=1-\sin \phi\right)$ and passive conditions (based on the Coulumb Theory), i.e. $k_{\mathrm{o}}$ and $k_{\mathrm{p}}$ conditions are gathered in the Figure 12.
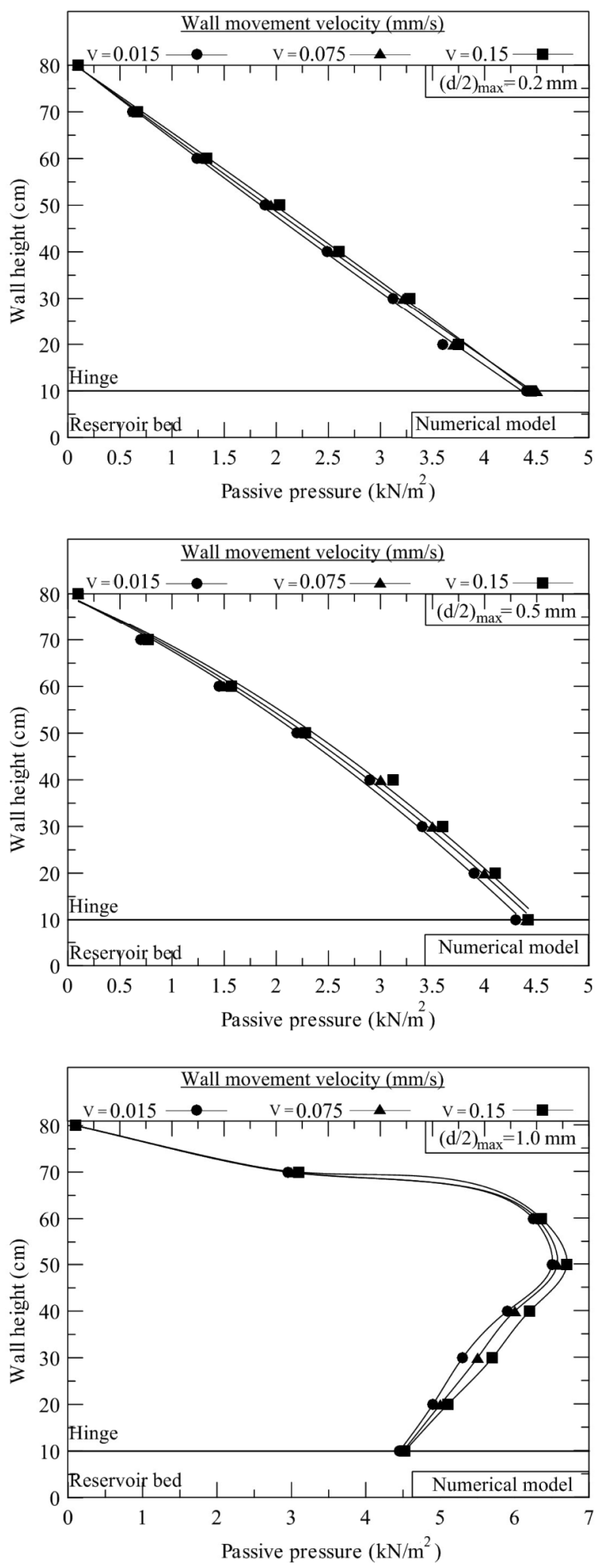

The following points can be deduced from the results comparison:

-For $(d / 2)_{\max }=0.2$ and $0.5 \mathrm{~mm}$ (namely small rotation), the passive pressure distribution is almost linear, and approximately coincides with at rest pressure distribution;
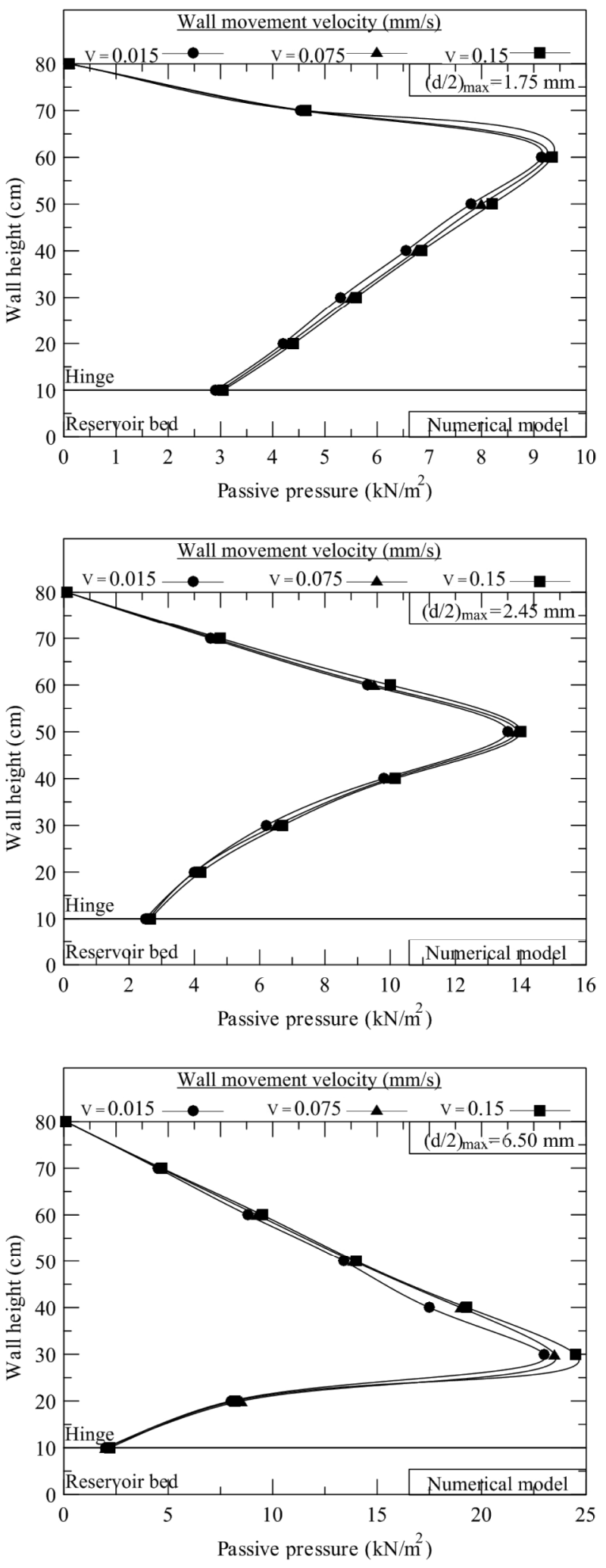

Fig. 9. The effect of the wall movement velocity on the passive earth pressure (numerical model) 

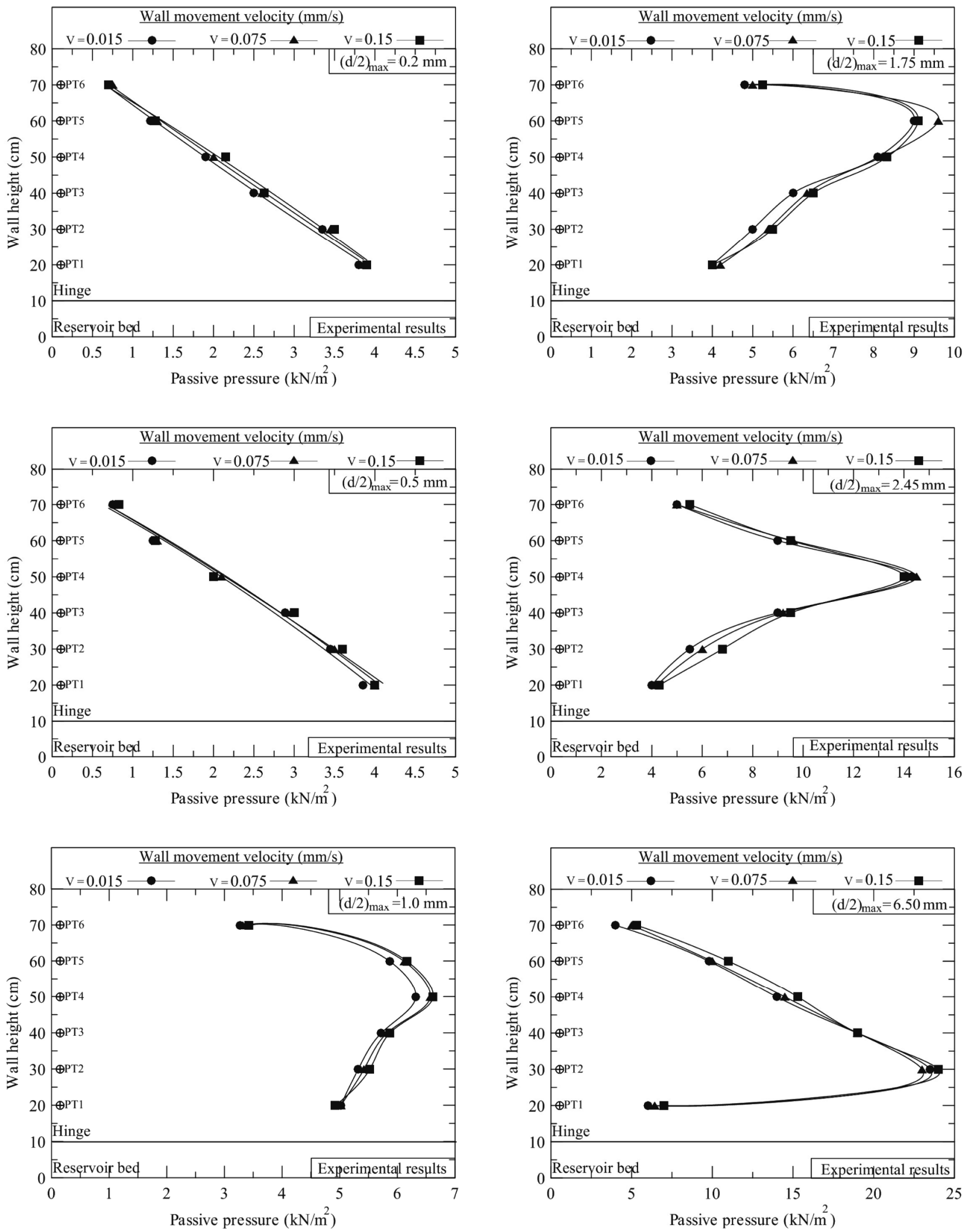

Fig. 10. The effect of the wall movement velocity on the passive earth pressure (experimental model) 


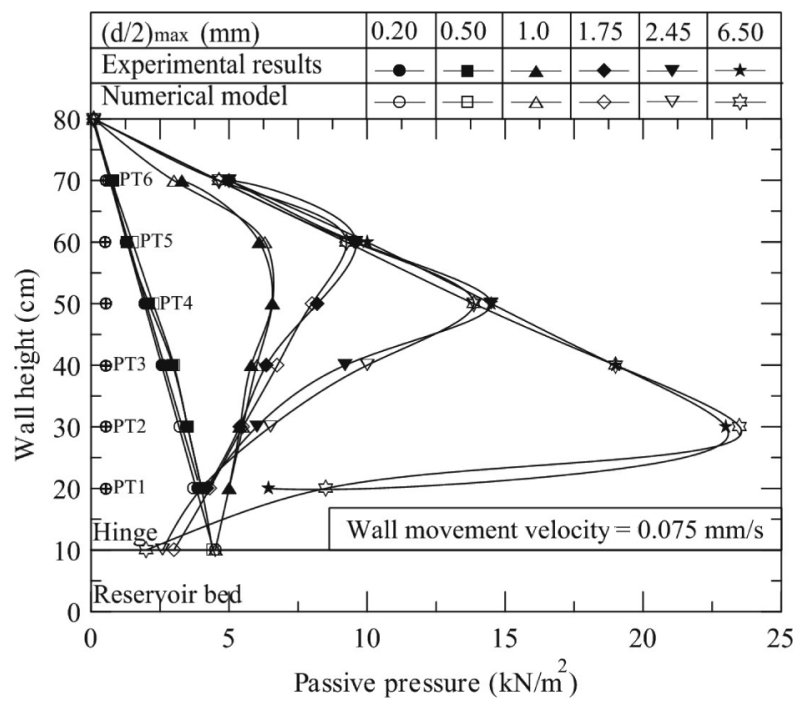

Fig. 11. Comparison between experimental and numerical model results in $0.075 \mathrm{~mm} / \mathrm{s}$ (the first cycle)

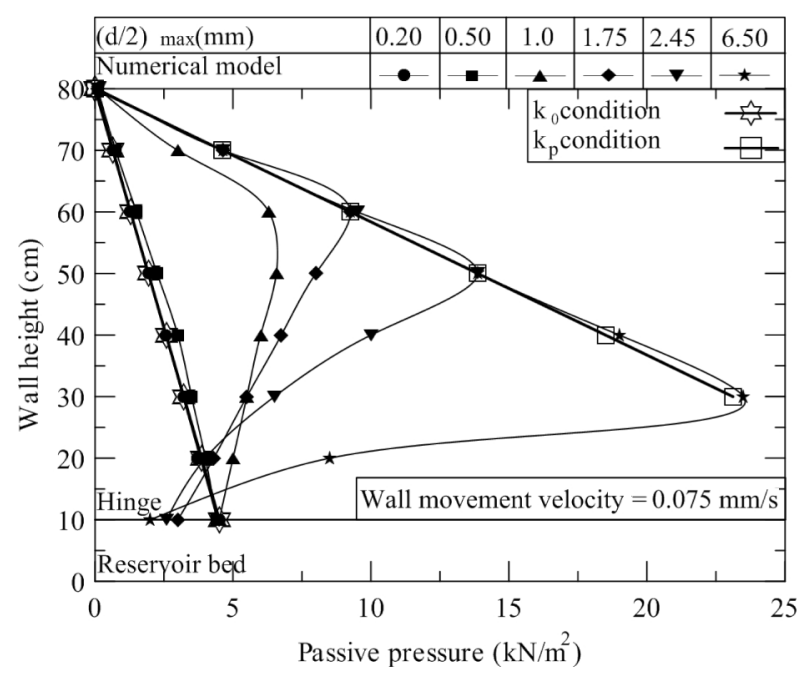

Fig. 12. A comparison between numerical model results with $k_{0}$ and $k_{\mathrm{p}}$ conditions

-With increasing wall rotation amplitude, the magnitude of passive pressure increases and its distribution changes from liner to non-linear regime. In addition, the location of maximum pressure moves gradually from the top of the wall towards the lower region. In this case, the passive pressure distribution can be divided two parts, above and below the maximum point;

-For $(d / 2)_{\max }=1 \mathrm{~mm}$ (namely medium rotation), distribution pressure in the above part lies between at rest condition and passive condition (based on the Coulumb Theory), and the below parts tends to decrease;

-For $(d / 2)_{\max }=1.75,2.45$ and $6.5 \mathrm{~mm}$ (namely large rotation), distribution of passive pressure in the above part coincides well with the passive condition (based on the Coulumb Theory). The below parts, however, tends to decrease and deviate gradually from the Coulumb Theory curve. This phenomenon suggests a different behavior of sand in these two parts. It seems in the above part, sand behaves as a plastic material, which is thought to be due to the large amplitude of wall displacement. A decline in passive pressure in the bottom part, however, is the result of forming arch between the wall and material (Tsang et al. 2002);

-It can be deduced that the magnitude of passive pressure is not constant and depends to the magnitude of wall rotation. In practical, it is not convenience to use Coulumb Theory for lateral earth pressure evaluation.

\subsection{Effect of number of cycles}

Finally, the influence of number of cycles on the lateral earth passive pressure is investigated. The results of the numerical model, is shown in Figure 13. The test was terminated when no significant changes in the magnitude and distribution regime of passive pressure were observed. The number of cycles, listed in Table 2.

The numerical results (Fig. 13) indicate a trend of the increase in passive pressure with increasing number of cycles. A comparison between the numerical and experimental results which are shown only for the $(d / 2)_{\max }=2.45$ and $6.5 \mathrm{~mm}$ (Fig. 14), indicates a good agreement between numerical analysis and test data.

The most important following points can be deduced from the results (Fig. 13):

- For small rotation of the wall $\left[(d / 2)_{\max }=0.2\right.$ and $0.5 \mathrm{~mm}$ ], the magnitude and regime of passive pressure are essentially independent of the number of cycles. This must be due to the elastic behavior of material and no destruction of the initial fabric of backfill material and no reduction in the initial voids ratio;

-For medium and large rotation of wall $\left[(d / 2)_{\max }=1\right.$, $1.75,2.45$ and $6.5 \mathrm{~mm}$ ], the magnitude of passive pressure increases with sequentially increasing number of cycles, but its rate decrease, so that after a limited number of cycles, the magnitude of passive pressure remains roughly constant. Additionally with increasing number of cycles, pressure on the bottom part of the wall and near the hinge decreases. This reduction in pressure is due to the increasing arch effect;

-Figure 12 clearly indicates that the magnitude of passive pressure in the first cycle lies between pressure at rest and passive condition. In addition, with increasing number of cycles the magnitude of passive pressure increases, i.e. the applied pressure to the wall maybe even becomes larger than passive pressure evaluated by Coulumb Theory.

\section{Estimation of arch zone}

As shown in Figure 12, when the wall is pushed inward to the fully passive condition, with the increase in the wall rotation amplitude, the passive pressure reaches to a maximum value after which decreases gradually. This clearly indicates a decrease in the surcharge, i.e. a decrease in 
vertical stress in the bottom part. This reduction is due to the forming arch between the wall and material, which results in the bottom the wall; lateral passive pressure decreases as well. If the wall starts to move back, formed arch most suddenly to be destroyed, and the vertical stress and horizontal pressure in turn, must be increased.

To investigate the process of arch formation, the variations of the passive pressure and different magnitudes of wall rotation are illustrated in Figure 15. In this section, the wall starts from at rest condition (vertical position), then wall is pushed inward to the fully passive condition $\left[(d / 2)_{\max }=0.2,0.5,1.0,1.75,2.45\right.$ and $\left.6.5 \mathrm{~mm}\right]$. It is then pulled back to the fully active condition and returned to the initial position. This is generally referred to one cycle. The data taken from six pressure transducers, PT1 to PT6 (Fig. 6) are shown in Figure 15. It should be noted that captured data are pertained to the first half of a cycle, i.e. from at rest to passive condition and just after retuning back.
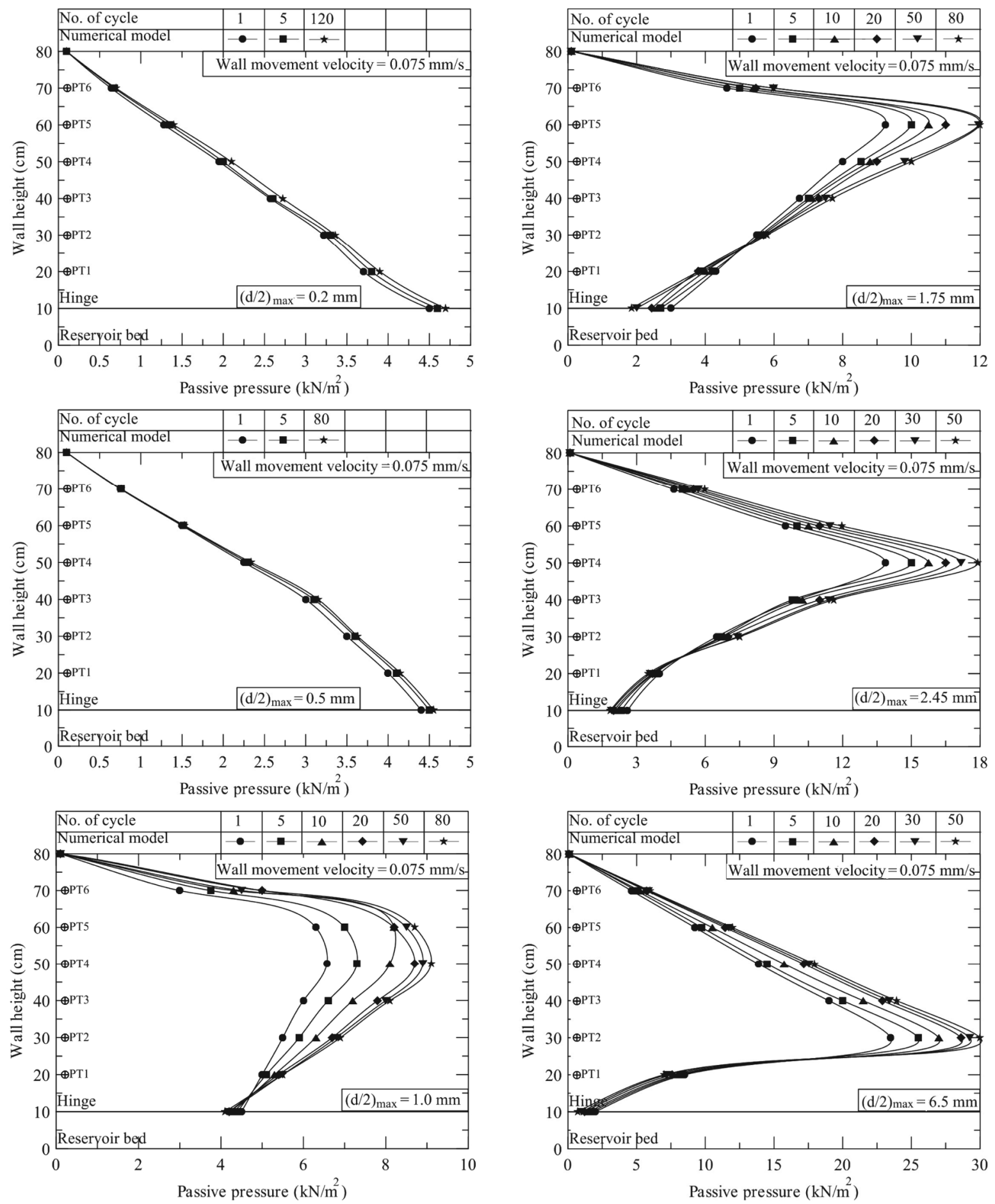

Fig. 13. The effect of number of cycles on the passive pressure (numerical model) 

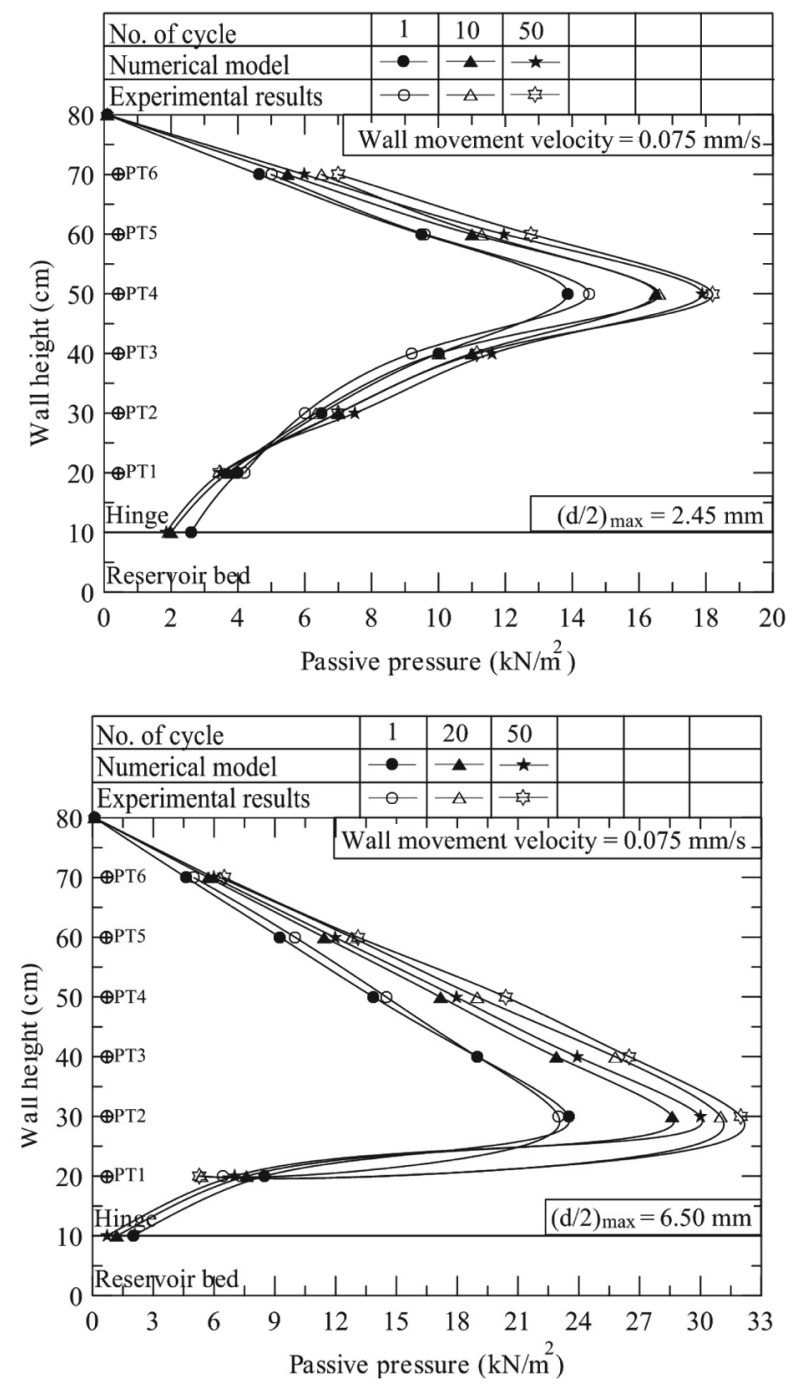

Fig. 14. Comparison between numerical and laboratory model results

The following points can be deduced from Figure 15:

-When the wall rotation is relatively small, say $(d / 2)_{\max }=0.2$ and $0.5 \mathrm{~mm}$, the soil behaves essentially elastic. The reason is that when the wall moves back, no significant change is observed in the induced lateral pressure, i.e. pressure remains at rest condition, and no passive condition is created;

- For medium rotation, say $(d / 2)_{\max }=1 \mathrm{~mm}$ (medium rotation), with moving back the wall, no significant increase in induced lateral pressure is observed. This indicates no arch has been formed (Fig. 15). However, it was previously observed (Fig. 12) that the pressure distribution has a maximum value, after which decreases gradually. This phenomenon will be discussed in more details later;

-For large rotation, say $(\mathrm{d} / 2)_{\max }=1.75,2.45$ and $6.5 \mathrm{~mm}$, the passive pressure distribution is similar to those for medium rotation. However, when the wall resumes to move back, pressure in transducers PT1 to PT4 [for $(\mathrm{d} / 2)_{\max }=1.75 \mathrm{~mm}$ ] and PT1 to PT3 [for $(\mathrm{d} / 2)_{\max }=2.45 \mathrm{~mm}$ ] and finally just PT1 [for $\left.(\mathrm{d} / 2)_{\max }=6.5 \mathrm{~mm}\right]$ is increased and no significant increase for the rest other pressure transducers is observed. This means that with moving back the wall, the forming arch is destroyed, which results in an increase in vertical stress. This, in turns, causes the lateral pressure to increase as well. In addition, it can be deduced that with increasing wall rotation amplitude, the height of the arch zone moves down. It is thought that if wall rotation increases further, (which is out of the working range of the laboratory model) fully passive condition is reached, i.e. the forming arch is (total) destroyed. It is necessary to mention that the results of some other cycles were similar to those of the first cycle; and this is the reason only the first cycle results are reported.

\section{Discussion}

The proposed numerical model in the Section 3 was again employed to clarify the experimental results. The elastic constitutive model (instead of the Mohr-Coulumb), however, was used to model the stress-strain behaviour of soils. The required parameters for this model are tabulated in Table $1(E, v)$. The magnitudes top of the wall were the same as for the laboratory test model, i.e. $(d / 2)_{\max }=0.2$, $0.5,1,1.75,2.45$ and $6.5 \mathrm{~mm}$. Figure 16 illustrates the numerical analysis results. In addition, included in this figure are the experimental results together with the pressure at rest and passive condition ( $\mathrm{k}_{\mathrm{o}}$ and $\mathrm{k}_{\mathrm{p}}$ respectively).

The most important points are as follows:

- For the wall rotation of $(\mathrm{d} / 2)_{\max }=0.2$ and $0.5 \mathrm{~mm}$, the pressure distribution for laboratory test is perfectly linear, and approximately equal to at rest pressure ( $\mathrm{k}_{\mathrm{o}}$ conditions);

- For the wall rotation of $(\mathrm{d} / 2)_{\max }=1 \mathrm{~mm}$, the induced pressure is totally non-liner. In this case, the pressure at the top region of the wall is less than passive condition. In the bottom region, however, the pressure distribution well coincides with elastic behaviour $\left[(d / 2)_{\max }=1 \mathrm{~mm}\right]$. This means that the behaviour of material in this region for this magnitude of wall rotation is elastic. In other words, the observed reduction in pressure is due to the elastic behaviour and not forming arch;

- For the wall rotation of $(\mathrm{d} / 2)_{\max }=1.75,2.45$ and $6.5 \mathrm{~mm}$, the induced pressure at the top region of the wall is tended to reach the passive pressure condition, but in the bottom region, it is not well coincided with elastic behavior $\left[(\mathrm{d} / 2)_{\max }=1.75,2.45\right.$ and $6.5 \mathrm{~mm}$ ]. In other words, the reduced pressure is mainly due to the arch effect.

\section{Conclusions}

The present research focuses mainly on the evaluation of interaction between soil and structure for integral bridge. A retaining wall laboratory model has been designed and constructed to imitate the cyclic behaviour of granular material behind bridge abutment. The research covers both experimental observations and numerical analysis results. The main conclusions of the research are outlined as follows: 
- The different velocity of the top of the wall movement in the range of this research has no significant effect on the induced lateral earth pressure. This means that the velocity of daily and seasonal movement (which are very low relatively to the selected range in this research) has no significant effects on the results;

-For medium and large rotation $\left[(d / 2)_{\max }=1,1.75\right.$, 2.45 and $6.5 \mathrm{~mm}$ ], with increasing wall rotation amplitude, the magnitude of passive pressure increases.
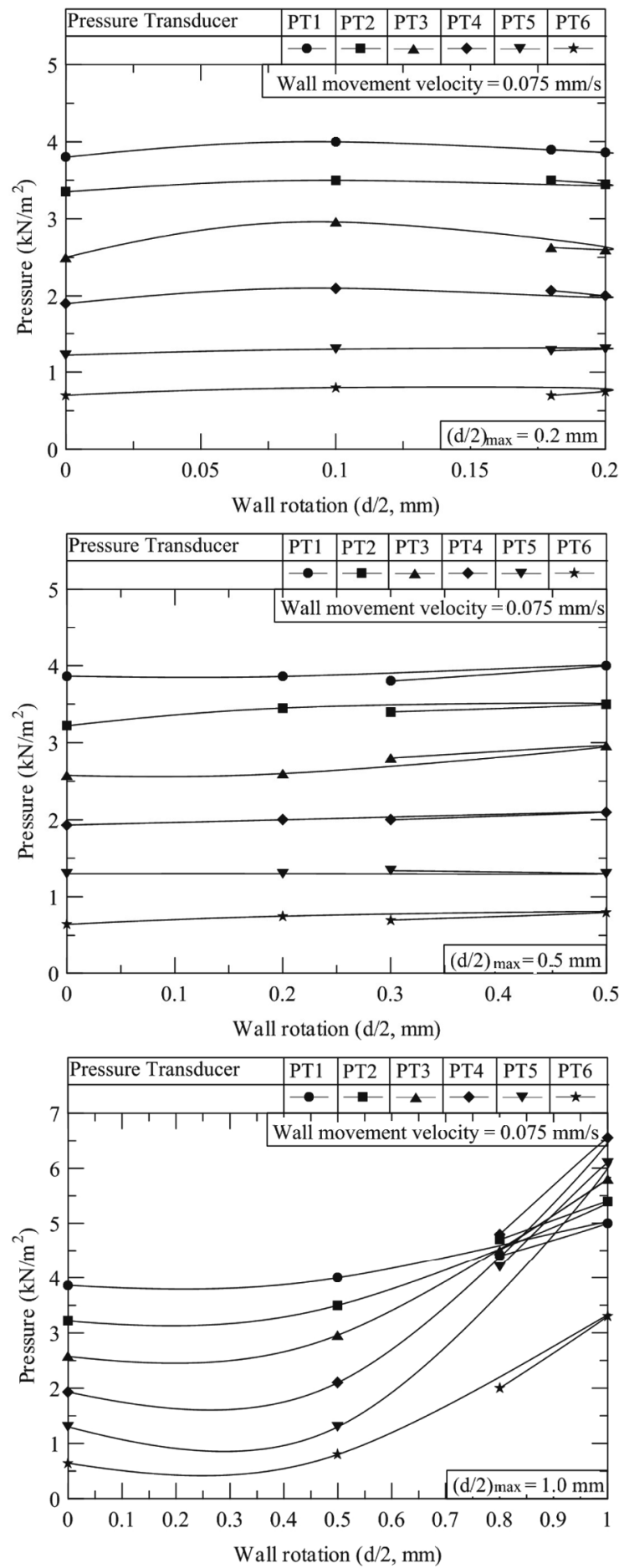

Fig. 15. Passive pressure measured at various depths
In addition, the location of maximum pressure moves gradually from the top of the wall towards the lower region;

-For medium and large rotation $\left[(d / 2)_{\max }=1,1.75\right.$, 2.45 and $6.5 \mathrm{~mm}$ ], the passive pressure increases with sequentially increasing number of cycles, but its rate decreases. Therefore, that after of a limited number of cycles the magnitude of passive pressure remains roughly constant;
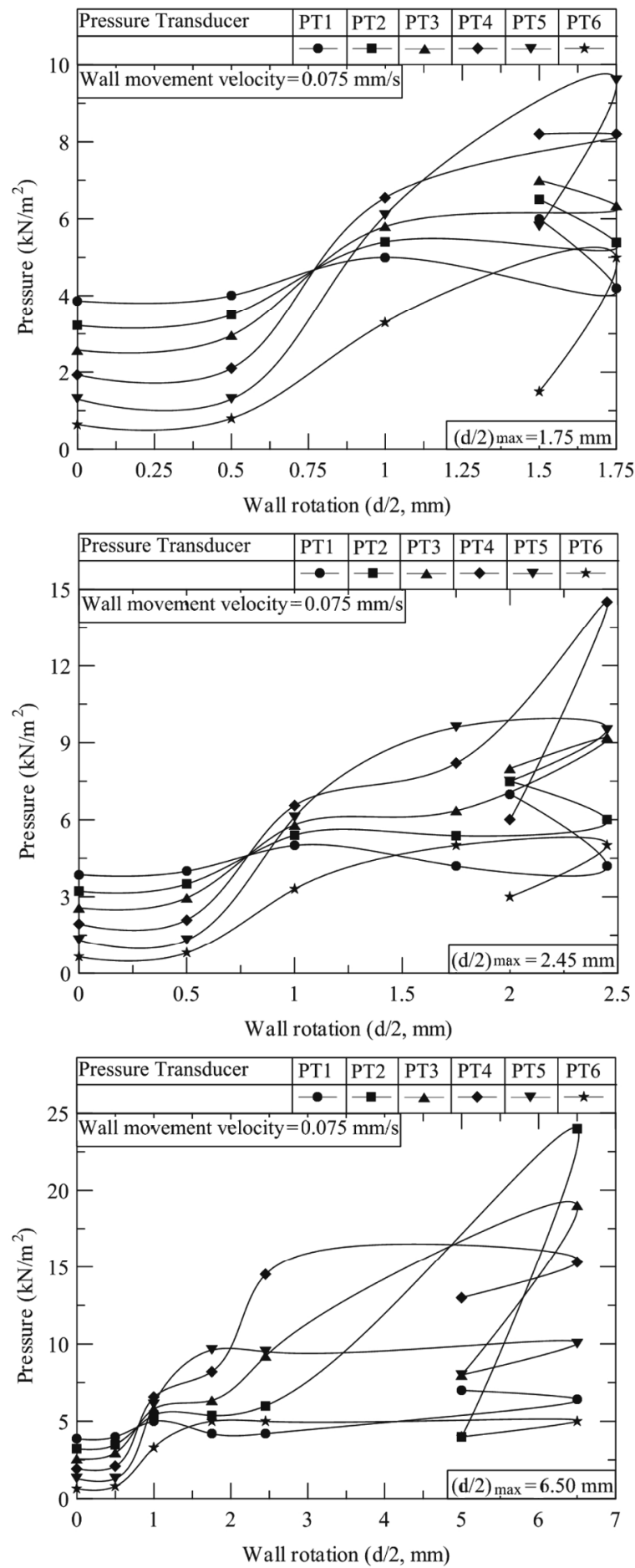


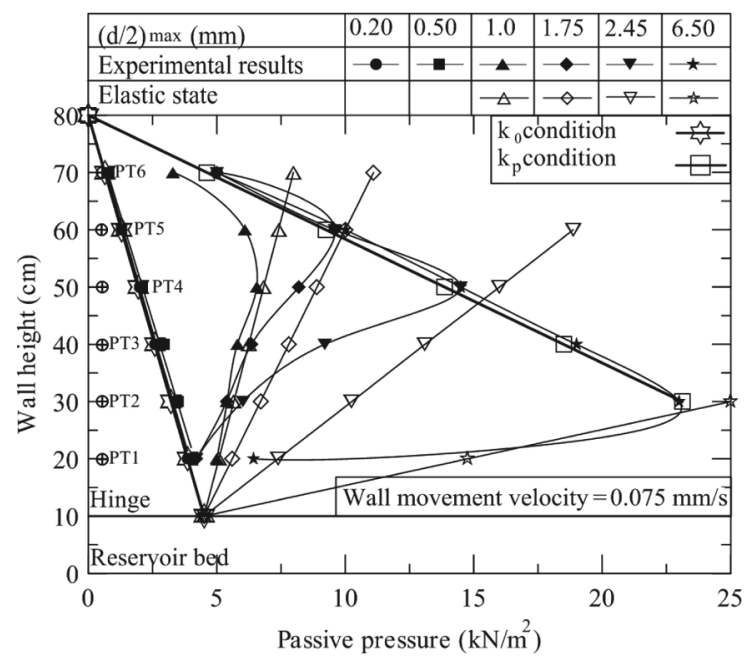

Fig. 16. A comparison between the numerical and experimental results with $k_{0}$ and $k_{\mathrm{p}}$ conditions

-For small rotation $\left[(d / 2)_{\max }=0.2,0.5 \mathrm{~mm}\right]$, the pressure distribution is almost linear, and approximately equal to the at rest pressure. However, with increasing wall rotation amplitude, in addition to pressure increase, the passive pressure distribution regime changes from liner to non-linear regime;

-For medium rotation $\left[(d / 2)_{\max }=1 \mathrm{~mm}\right]$, the induced pressure on the top region of the wall lies between pressure at rest and passive condition. In the bottom region, the pressure gradually decreases. This reduction in pressure is thought to be due to the elastic behavior of material and not arch phenomena;

-For large rotation $\left[(d / 2)_{\max }=1.75,2.45\right.$ and $6.5 \mathrm{~mm}$ ], the induced pressure on the top region of the wall matches well with passive condition and in the bottom region the pressure gradually decreases, which is mainly due to the arch formation;

-For large rotation $\left[(d / 2)_{\max }=1.75,2.45\right.$ and $6.5 \mathrm{~mm}$, with increasing number of cycles and amplitude, pressure on the bottom part of the wall and near the hinge decreases. This is due to the increasing effect of arching formation, i.e. the forming arch becomes stiffer and stiffer during success cycles;

-With increasing wall rotation amplitude, the magnitude of passive pressure increases. In addition, the location of maximum pressure moves gradually from the top of the wall towards the lower region. It can be deduced that with increasing wall movement amplitude, the height of the arch zone moves down;

-Finally, it can be concluded that the magnitude of passive pressure in integral bridge abutment is very different from that evaluated by Coulumb Theory. For bridges with small wall rotation, the induced pressure is in the range of at-rest condition. For large rotation, however it may exceed from passive pressure based on the Coulumb Theory. This means that the design of this bridge with classic theory leads to overestimate or underestimate design, which is sometimes high risk in these projects.

\section{References}

Ahn, J.-H.; Yoon, J.-H.; Kim, J.-H.; Kim, S.-H. 2011. Evaluation on the behavior of abutment-pile connection in integral abutment bridge, Journal of Constructional Steel Research 67(7): 1134-1148. http://dx.doi.org/10.1016/j.jcsr.2011.02.007

Alizadeh, M. H.; Rashid, A. R. K.; Chik, Z.; Mirhosseiny, S. M. 2010. Investigation of abutment displacement of a full height integral bridges in dense granule backfill, American Journal of Engineering and Applied Sciences 3(4): 749-756. http://dx.doi.org/10.3844/ajeassp.2010.749.756

Arsoy, S. 2000. Experimental and analytical investigation of piles and abutment bridges: $\mathrm{PhD}$ Thesis. Virginia Polytechnic Institute and State University.

Banks, J. R.; Knight, T.; Young, J.; Bloodworth, A. G. 2008. Modeling granular soil to predict pressures on integral bridge abutments, in Proc. of Fourth International Conference on Bridge Maintenance, Safety and Management, 13-17 July, 2001, Seoul, Korea. 8 p.

Barker, K. J.; Carder, D. R. 2000. Performance of the two integral bridges forming the A62 Manchester road overbridge. Berkshire: Crowthorne. $38 \mathrm{p}$.

Barker, K. J.; Carder, D. R. 2001. Performance of an integral bridge over the M1-Al Link Road at Bramham crossroads. Berkshire: Crowthorne. $42 \mathrm{p}$.

Bolton, M. D. 1986. The strength and dilatancy of sands, Journal of Géotechnique 36(1): 65-78. http://dx.doi.org/10.1680/geot.1986.36.1.65

Broms, B. B.; Ingleson, I. 1971. Earth pressure against the abutments of a rigid frame bridge, Journal of Geotechnique 21(1): 15-28. http://dx.doi.org/10.1680/geot.1971.21.1.15

Broms, B. B.; Ingleson, I. 1972. Lateral Earth pressure on a bridge abutment, in Proc. of the $5^{\text {th }}$ European Conference on Soil Mechanics and Foundation Engineering, 10-13 April, 1972, Madrid, 117-123.

Card, G. B.; Carder, D. R. 1993. A literature review of the geotechnical aspects of integral bridge abutments. Berkshire: Crowthorne. $63 \mathrm{p}$.

Carlstedt, E. 2008. Soil-structure interaction for bridges with backwalls: Master of Science Thesis. Structural Design and Bridges, Stockholm, Sweden.

Charuchaimontri, T.; Senjuntichai, T.; Ozbolt, J.; Limsuwan, E. 2008. Effect of lap reinforcement in link slabs of highway bridges, Engineering Structures 30(2): 546-560. http://dx.doi.org/10.1016/j.engstruct.2007.04.015

Chen, C. N.; Huang, W.-Y.; Tseng, C.-T. 2011. Stress redistribution and ground arch development during tunneling, Tunnelling and Underground Space Technology 26(1): 228-235. http://dx.doi.org/10.1016/j.tust.2010.06.012

Darley, P.; Carder, D. R.; Barker, K. J. 1998. Seasonal thermal effects over three years on the shallow abutments of an integral bridge in Glasgow. Berkshire: Crowthorne. $81 \mathrm{p}$.

Das, B. M. 2002. Principals of foundation engineering. $4^{\text {th }}$ ed. Bill Stenquist, Virginia, USA.

Dicleli, M. 2000. A rational design approach for prestressedconcrete-girder integral bridges, Engineering Structures 22(3): 230-245. http://dx.doi.org/10.1016/S01410296(98)00080-7

Dicleli, M.; Albhaisi, S. M. 2004. Performance of abutmentbackfill system under thermal variations in integral bridges built on clay, Engineering Structures 26(7): 949-962. http://dx.doi.org/10.1016/j.engstruct.2004.02.014 
Dicleli, M.; Erhan, S. 2010. Effect of soil-bridge interaction on the magnitude of internal forces in integral abutment bridge components due to live load effects, Engineering Structures 32(1): 129-145.

http://dx.doi.org/10.1016/j.engstruct.2009.09.001

Duncan, J. M.; Arsoy, S. 2003. Effect of bridge-soil interaction on behavior of piles supporting integral bridge. Washington, D.C: TRB. 24 p.

Emerson, M. 1977. Temperatures differences basis of design requirements. Berkshire: Crowthorne. $39 \mathrm{p}$.

England, G. L.; Bolouri-Bazaz, J. 1995. Ratching flow of granular materials, in Proc. of Static and Dynamic Properties of Gravelly Soil, AISC, Geotechnical Division, 23-27 October, 1995, San Diego, California, US, 117-125.

England, G. L.; Tsang, N. C. M.; Bush, D. L. 2000. Integral bridges: a fundamental approach to the time-temperature loading problem. London: Thomas Telford. $152 \mathrm{p}$. http://dx.doi.org/10.1680/ibafattttlp.35416

Erhan, S.; Dicleli, M. 2009. Live load distribution equations for integral bridge substructures, Engineering Structures 31(5): 1250-1264. http://dx.doi.org/10.1016/j.engstruct.2009.01.020

Fang, Y. S.; Chen, T. J.; Wu, B. F. 1994. Passive earth pressures with various wall movements, Journal of Geotechnical Engineering 120(8): 1307-1323. http://dx.doi.org/10.1061/(ASCE)07339410(1994)120:8(1307)

Fang, Y. S.; Ho, Y. C.; Chen, T. J. 2002. Passive earth pressure with critical state concept, Journal of Geotechnical and Geo-environmental Engineering 128(8): 651-659.

http://dx.doi.org/10.1061/(ASCE)10900241(2002)128:8(651)

Fang, Y. S.; Ishibashi, I. 1986. Static earth pressures with various wall movements, Journal of Geotechnical Engineering 112(3): 317-333.

http://dx.doi.org/10.1061/(ASCE)07339410(1986)112:3(317)

Hoppe, E. J.; Gomez, J. P. 1996. Field study of an integral back wall bridge. Virginia: Charlottesville. $47 \mathrm{p}$.

Horvath, J. S. 2000. Integral abutment bridge: problem and innovative solutions using EPS geofoam and other geosynthetics. New York: Bronx. $170 \mathrm{p}$.

James, R. G.; Bransby, P. L. 1970. Experimental and theoretical investigations of a passive earth pressure problem, Journal of Geotechnique 20(1): 17-37. http://dx.doi.org/10.1680/geot.1970.20.1.17

Khodair, Y. A.; Hassiotis, S. 2005. Analysis of soil-pile interaction in integral abutment, Journal of Computers and Geotechnics 32(3): 201-209.

http://dx.doi.org/10.1016/j.compgeo.2005.01.005

Kim, W.; Laman, J. A. 2010a. Integral abutment bridge response under thermal loading, Engineering Structures 32(6): 1495-1508.

http://dx.doi.org/10.1016/j.engstruct.2010.01.004
Kim, W.; Laman, J. A. 2010b. Numerical analysis method for long-term behavior of integral abutment bridges, Engineering Structures 32(8): 2247-2257.

http://dx.doi.org/10.1016/j.engstruct.2010.03.027

Lock, R. J. 2002. Integral bridge abutments. London: Arup, 13 Fitzroy Street. $50 \mathrm{p}$.

Marques Lima, J.; De Brito, J. 2009. Inspection survey of 150 expansion joints in road bridges, Engineering Structures 31(5): 1077-1084. http://dx.doi.org/10.1016/j.engstruct.2009.01.011

Noorzaei, J.; Abdulrazeg, A. A.; Jaafar, M. S.; Kohnehpooshi, O. 2010. Non-linear analysis of an integral bridge, Journal of Civil Engineering and Management 16(3): 387-394. http://dx.doi.org/10.3846/jcem.2010.44

Olmo Segovia, A. 2006. FEM simulation of a steel box culvert test - comparisons of numerical results with field data: Master of Science Thesis. Royal Institute of Techniligy (KTH), Stockholm, Sweden.

Paik, K. H.; Salgado, R. 2003. Estimation of active earth pressure against rigid retaining walls considering arching effects, Journal of Geotechnique 53(7): 643-653. http://dx.doi.org/10.1680/geot.2003.53.7.643

Phillip, S. K. O. P. E.; Xiaobin, L.; Harold, S. H .P. E. 2010. Numerical study of an integral abutment bridge supported on drilled shafts, Journal of Bridge Engineering 15(1): 19-31.

http://dx.doi.org/10.1061/(ASCE)BE.1943-5592. 0000037

Potts, D. M.; Zdravkovic', L. 2001. Finite element analysis in geotechnical engineering. London: Thomas Telford. http://dx.doi.org/10.1680/feaigea.27831

Roberts, A. W. 2010. Review of mass-flow hopper design with respect to stress fields and surcharge loads, Particuology 8(6): 591-594. http://dx.doi.org/10.1016/j.partic.2010.07.016

Sanford, T. C.; Elgaaly, M. 1993. Skew effects on backfill pressures at frame bridge abutmebts. Washington, D.C: TRB. 11 p.

Smith, I. M.; Griffiths, D. V. 1998. Programming the finite element method. New York: John Wiley \& Sons. 482 p.

Springman, S .M.; Norrish, A. R. M.; Ng, C. W. 1996. Cyclic loading of sand behind integral bridge abutment. Berkshire: Crowthorne. $69 \mathrm{p}$.

Thomson, T. A. J. 1999. Passive earth pressures behind integral bridge abutments: PhD Thesis. University of Massachusetts Amherst.

Tsang, N. C. M.; England, G.; Dunstan, T. 2002. Soil/structure interaction of integral bridge with full height abutments, in Proc. $15^{\text {th }}$ ASCE Engineering Mechanics Conference, Columbia University, New York, NY, USA.

Wallbank, E. J. 1989. The performance of concrete in bridge: a survey of 200 Highway Bridges, London: HMSO. 96 p.

Mojtaba MOVAHEDIFAR, at the Department of Civil Engineering, Science and Research Branch, Islamic Azad University, Tehran, IRAN. He has been a PhD candidate, in Geotechnical engineering, since 2006, in Science and Research Branch, Islamic Azad University. He finished his MSc in Geotechnical engineering, Ferdowsi University, 1998-2001. His main research interests include soil-structure interaction, integral bridge abutment, retaining wall.

Jafar BOLOURI-BAZAZ, at Civil Engineering Department, Ferdowsi University of Mashhad, Mashhad, IRAN. He finished his PhD in Geotechnique at Imperial College of Science and Technology, London University, in 1994-1999 and MSc in Structural Engineering, at Tarbiat Modarres University, Tehran, Iran, in 1991-1994. He was an Associate Professor at the Civil Engineering Department, Ferdowsi University of Mashhad, 1990-2011. His main research interests include soil improvement, soil dynamics, foundation engineering, excavation and tunnelling. 